\title{
Exactly two-to-one maps from continua onto some tree-like continua
}

\author{
by
}

\author{
W. Dębski (Katowice), J. Heath (Auburn, Ala.) \\ and J. Mioduszewski (Katowice)
}

\begin{abstract}
It is known that no dendrite (Gottschalk 1947) and no hereditarily indecomposable tree-like continuum (J. Heath 1991) can be the image of a continuum under an exactly 2-to-1 (continuous) map. This paper enlarges the class of tree-like continua satisfying this property, namely to include those tree-like continua whose nondegenerate proper subcontinua are arcs. This includes all Knaster continua and Ingram continua. The conjecture that all tree-like continua have this property, stated by S. Nadler Jr. and L. E. Ward Jr. (1983), is still neither confirmed nor rejected.
\end{abstract}

1. Introduction. A map means throughout this paper a continuous map. A map is 2-to-1 if it assumes each value exactly twice.

We consider tree-like continua whose nondegenerate proper subcontinua are arcs. The best known of these is the Knaster continuum that results when the inverse elements, $x$ and $-x$, are identified in the dyadic solenoid. Even for this clearly understood continuum it was not known until now if it is a 2-to- 1 image of a continuum.

All of the Knaster continua, one of which is the Knaster continuum, are known to be arc-like and to have only arcs as nondegenerate proper subcontinua. There are examples of such continua that are tree-like but not arc-like, for instance the Ingram continua [6].

Our main result in this paper is the theorem asserting that tree-like continua whose nondegenerate proper subcontinua are arcs cannot be 2-to-1 images of continua. This can be extended to some finite unions of such continua in the final notes.

Recall that a continuum is tree-like if, for every $\varepsilon>0$, it admits a map

1991 Mathematics Subject Classification: Primary 54C10.

Key words and phrases: Knaster continua, Ingram continua, 2-to-1 map.

Research of the first-named and third-named authors supported by scientific grant R.P.I.10. 
onto a (finite) tree such that each point inverse has diameter less than $\varepsilon$. If this can be done with arcs, the continuum in question is called arc-like. This can be translated into the language of inverse limits of finite trees. The Knaster continua are distinguished by the property that the bonding maps between arcs in the sequence are open.

We shall use the following property of maps that follows from the Baire category theorem, namely that if $f$ is a continuous map from the compactum $X$ onto a compactum $Y$ then almost all, in the sense of Baire category, points $y$ of $Y$ are points of openness of $f$, which means that if $f(x)=y$, then $y$ is in the interior of $f(U)$ for any neighborhood $U$ of $x$. If the map is 2-to-1 (or $k$-to-1), then these points form an open subset of $Y$.

2. Preliminary lemmas. Suppose that $Y$ is a metric continuum whose nondegenerate proper subcontinua are arcs. If $Y$ is decomposable, then $Y$ is the union of two arcs and thus is an arc or a simple closed curve. If $Y$ is indecomposable, then each composant of $Y$ is an increasing union of arcs and is either a continuous 1-to-1 image of an open ray, or a continuous 1-to-1 image of an open line.

Suppose that $Y$ is indecomposable and $N$ is an arc in $Y$. Remove the geometric interior of $N$ from the arc component of $Y$ in which $N$ lies. The remainder of the arc component splits into two arcwise connected sets, at least one of which, say $E$, is dense in $Y$. The set $E$ is a continuous 1-to-1 image of the closed half line. The endpoint common to $E$ and $N$ will be called a free endpoint of $N$. Each arc in $Y$ has at least one free endpoint.

The decomposable cases - i.e. the arc and the circle - are not considered here, since the circle is not tree-like and the arc falls within the scope of Gottschalk's [2] theorem. So, we shall assume the indecomposability of $Y$. In the two lemmas of this section the hypothesis of tree-likeness will not be used, so the lemmas of this section are valid for solenoids, for instance.

LEMMA 1. If $Y$ is an indecomposable continuum whose nondegenerate proper subcontinua are arcs, $f$ is a map from a continuum $X$ onto $Y$, $L$ is an arc in $Y$, and $C$ is a component of $f^{-1}(L)$, then $f(C)$ contains an endpoint of $L$ that is not the endpoint of the composant of $Y$ containing $L$.

Proof. Suppose the lemma fails and that no endpoint of $L$ is in $f(C)$ unless it is also the endpoint of the composant of $Y$ which contains $L$. Take an open subset $U$ of $Y$ containing $f(C)$ such that the closure of $U$ contains the same ends of $L$ as $f(C)$. The component $C^{\prime}$ of $f^{-1}(U)$ that contains $C$ must go to the boundary of $f^{-1}(U)$, by the Janiszewski Lemma, so the closure of $C^{\prime}$ is a continuum that contains $C$ properly. But the closure of $C^{\prime}$ maps into $L$ under $f$ since its image intersects $L$ but contains no endpoint 
of $L$ unless it is also the endpoint of the composant. This contradicts the fact that $C$ is a component of $f^{-1}(L)$.

Lemma 2. Suppose $Y$ is an indecomposable continuum whose nondegenerate proper subcontinua are arcs, $f$ is a 2-to-1 map from a continuum $X$ onto $Y, L$ is an arc in $Y$ from $t$ to $x$, and $C$ is a component of $f^{-1}(L)$ such that $t \notin f(C)$. Let $N$ be an extension of $L$ through $x$, i.e. an arc with endpoint $t$ containing $L$. Then there is a component $D$ of $f^{-1}(N)$ for which we still have $t \notin f(D)$.

Proof. By Lemma 1, $x$ belongs to $f(C)$. Let $M$ denote the subarc of $N$ from $x$ to $y$, where $y$ is the endpoint of $N$ different from $t$, and let $B$ denote the component of $f^{-1}(M)$ that intersects $C$. If $B$ contains both points that map to $x$ (recall that $f$ is 2-to- 1 ), then any other component $D$ of $f^{-1}(M)$ is a component of $f^{-1}(N)$ such that $t \notin f(D)$. The existence of another component follows from Gottschalk's result [2]. If $B$ contains only one point that maps to $x$ under $f$, then the component $D$ of $f^{-1}(N)$ containing $C$ (and $B$ ) satisfies $t \notin f(D)$.

LEMMA 3. If $Y$ is an indecomposable continuum whose nondegenerate proper subcontinua are arcs, $f$ is a 2-to-1 map from a continuum $X$ onto $Y$, $L$ is an arc in $Y$ and $C$ is a component of $f^{-1}(L)$, then $f(C)$ contains a free endpoint of $L$.

Proof. It was proved in the preliminary discussion that at least one of the endpoints of $L$ is free. Let $x$ and $t$ denote the endpoints of $L$. By Lemma 1 , we know that $f(C)$ contains at least one endpoint of $L$. So it remains to exclude the case when $f(C)$ contains $x$ but not $t, x$ is not free and $t$ is free. Since $x$ is not free, there is another arc $M$ intersecting $L$ in exactly the one endpoint $x$ such that the other endpoint $y$ of $M$ is the endpoint of the composant containing $L$. By Lemma 2 there is a component $K$ of $f^{-1}(L \cup M)$ such that $t \notin f(K)$. This contradicts Lemma 1.

3. Further lemmas. In this section the hypothesis that $Y$ is tree-like will be used in the lemmas.

Grispolakis and Tymchatyn [3] showed that every map from a continuum onto an atriodic tree-like continuum is weakly confluent, i.e. the inverse images of subcontinua contain components which are mapped onto these subcontinua. So, we get:

LEMMA 4. If $Y$ is a tree-like continuum whose nondegenerate proper subcontinua are arcs, $f$ is a map from a continuum onto $Y$, and $L$ is an arc in $Y$, then some component of $f^{-1}(L)$ maps onto $L$.

LEMma 5. Let $g$ be a map from an arc E into a tree $T$. Let $T^{\prime}$ be a subtree of $T$ such that the values of $g$ at the endpoints of $E$ belong to $T^{\prime}$. Then, for 
each complementary interval $W$ of $E \backslash g^{-1}\left(T^{\prime}\right)$ in $E$, the map g assumes the same values at the endpoints of $W$.

Proof. Let $a$ and $b$ be the endpoints of $W$. Then $g(\bar{W}) \cap T^{\prime}=$ $\{g(a), g(b)\}$. On the other hand, the set $g(\bar{W}) \cap T^{\prime}$, being the intersection of two subcontinua of the tree $T$, must be connected. Thus $g(a)=g(b)$.

A compactum is $\delta$-connected if $\delta$ is a positive number and the compactum cannot be written as the union of two disjoint compacta whose distance apart is more than $\delta$.

LemMa 6. Suppose $Y$ is a compact space having only arcs as its proper nondegenerate subcontinua. Let $A$ be an arc in $Y$ and let $\varepsilon>0$ be given. Let $D$ be an open subset of $Y$ such that $A$ has both endpoints in D. Then there exists a $\delta>0$ such that if $S$ is a $\delta$-connected subcompactum of $Y$ contained in the $\delta$-neighborhood of $Y \backslash D$ and intersecting the $\delta$-neighborhood of $A$, then $S$ lies in the $\varepsilon$-neighborhood of $A$.

P r o of. Note that the components of $A \backslash D$ are components of $Y \backslash D$. This follows from the fact that $A$ has both endpoints in $D$ and from the assumptions on $Y$. Since the components and the quasi-components of $Y \backslash D$ coincide, there exists a closed-open subset $F$ of $Y \backslash D$ containing $A \backslash D$ and lying in the $\varepsilon$-neighborhood of $A$. Let $\delta$ be less than a third of the distance between the disjoint compact sets $F \cup A$ and $(Y \backslash D) \backslash F$, and such that the $\delta$-neighborhood of $F$ is contained in the $\varepsilon$-neighborhood of $A$. Now, if $S$ is a $\delta$-connected compactum contained in the $\delta$-neighborhood of $Y \backslash D$, then $S$ is contained entirely in the $\delta$-neighborhood of $F$ or in the $\delta$-neighborhood of $(Y \backslash D) \backslash F$, since the sets $F$ and $(Y \backslash D) \backslash F$ have distance greater than $3 \delta$. So, if the compactum $S$ has points in the $\delta$-neighborhood of $A$, then $S$ lies in the $\delta$-neighborhood of $F$, and thus in the $\varepsilon$-neighborhood of $A$.

Suppose $\varepsilon$ is a positive number and the $\operatorname{arcs} C$ and $A$ lie in the same oriented composant of an indecomposable continuum whose nondegenerate proper subcontinua are arcs. We say that the arc $C$ has reverse orientation within $\varepsilon$ of $A$ if $C$ lies in the $\varepsilon$-neighborhood of $A$ and the $\varepsilon$-neighborhood of the last point of $A$ contains the first point of $C$ and the $\varepsilon$-neighborhood of the first point of $A$ contains the last point of $C$.

LEMMA 7. Let $Y$ be an indecomposable tree-like continuum such that each proper nondegenerate subcontinuum of $Y$ is an arc. Let $A$ be an arc in $Y$ and $\varepsilon$ be a positive number. Then there is an arc $C$ in the same composant as $A$ with reverse orientation within $\varepsilon$ of $A$.

Proof. Let $U$ and $V$ denote closure disjoint open sets, $U$ about the first point $x$ and $V$ about the last point $y$ of $A$, each having diameters less than $\varepsilon$. Let $\delta>0$ be so small that 
(1) the $\delta$-neighborhood of $x$ is contained in $U$ and the $\delta$-neighborhood of $y$ is contained in $V$,

(2) $\delta$ is less than the distance between $\bar{U}$ and $\bar{V}$,

(3) $\bar{U}$ is contained in the $(\varepsilon-\delta)$-neighborhood of $x$, and $\bar{V}$ is contained in the $(\varepsilon-\delta)$-neighborhood of $y$, and

(4) if $S$ is a $\delta$-connected compactum contained in the $\delta$-neighborhood of $Y \backslash(U \cup V)$ and intersecting the $\delta$-neighborhood of $A$, then $S$ lies in the $\varepsilon$-neighborhood of $A$.

The existence of a $\delta$ satisfying (4) is assured by Lemma 6 . Since $Y$ is tree-like there is a $\delta$-map, $g$, from $Y$ onto a tree $T$. From (1) it follows that $g^{-1}(g(x)) \subset U$ and $g^{-1}(g(y)) \subset V$, and, in consequence, that $g(x) \in$ $\operatorname{int} g(\bar{U})$ and $g(y) \in \operatorname{int} g(\bar{V})$. From (2) it follows that $g(\bar{U}) \cap g(\bar{V})=\emptyset$.

Let $B$ be the arc from $g(x)$ to $g(y)$. Since $Y$ is indecomposable and the composants of $Y$ are dense, we may assume that the subset of the $A$ composant consisting of points past $y$ is dense in $Y$. Let $x^{\prime}$ be the first point past $y$ in the $A$-composant such that $g\left(x^{\prime}\right) \in B \cap g(\bar{U})$. Let $y^{\prime}$ be the last point past $y$, but before $x^{\prime}$, in the $A$-composant such that $g\left(y^{\prime}\right) \in B \cap g(\bar{V})$; such points exist because $g(x) \in \operatorname{int} g(\bar{U})$ and $g(y) \in B \cap \operatorname{int} g(\bar{V})$. Denote by $E$ the arc joining $x^{\prime}$ and $y^{\prime}$.

Let $T^{\prime}$ be the closure of the component of $g(E) \backslash(g(\bar{U}) \cup g(\bar{V}))$ containing the arc joining $g\left(x^{\prime}\right)$ and $g\left(y^{\prime}\right)$ in $T$. The set $S=E \cap g^{-1}\left(T^{\prime}\right)$ is contained in the $\delta$-neighborhood of $Y \backslash(U \cup V)$. We have $T^{\prime} \subset g(E)$ and therefore $S$ is $\delta$-connected, since $g$ restricted to $E$ is a $\delta$-map. Moreover, $S$ has points in the $\delta$-neighborhood of $A$. To see this, observe that $g(A) \cap T^{\prime} \neq \emptyset$, as the arc from $g\left(x^{\prime}\right)$ to $g\left(y^{\prime}\right)$ is contained in $T^{\prime} \cap g(A)$. Now, if $t \in g(A) \cap T^{\prime}$, then $g^{-1}(t) \cap A \neq \emptyset$ and $g^{-1}(t) \cap S \neq \emptyset$. Since the diameter of $g^{-1}(t)$ is less than $\delta$, it follows that $S$ has points in the $\delta$-neighborhood of $A$. Hence, by (4), $S$ lies in the $\varepsilon$-neighborhood of $A$.

By the definition of $T^{\prime}$, we have $g\left(x^{\prime}\right) \in T^{\prime}$ and $g\left(y^{\prime}\right) \in T^{\prime}, x^{\prime}$ and $y^{\prime}$ being the endpoints of $E$. Applying Lemma 5, we infer that $g$ has the same values, belonging both to $g(\bar{U})$ or both to $g(\bar{V})$, at the endpoints of the intervals of $E$ complementary to $g^{-1}\left(T^{\prime}\right)$. Since the value $g\left(y^{\prime}\right)$, at the first endpoint $y^{\prime}$ of $E$, lies in $g(\bar{V})$, and the value $g\left(x^{\prime}\right)$, at the last endpoint $x^{\prime}$ of $E$, lies in $g(\bar{U})$, there exists a component of $S$ such that $g$ has a value in $g(\bar{V})$ at the first endpoint and in $g(\bar{U})$ at the last endpoint of this component. This component is the desired arc $C$. The first endpoint of $C$ lies in the $\varepsilon$-neighborhood of $y$ and the last endpoint lies in the $\varepsilon$-neighborhood of $x$; this is a consequence of $(3)$ as $g$ is a $\delta$-map. The arc $C$ lies in $S$, and thus in the $\varepsilon$-neighborhood of $A$.

Note. If $T$ is an arc (the case, for instance, when $Y$ is arc-like), we have $T^{\prime}=g(E)$. In consequence, $g^{-1}\left(T^{\prime}\right) \cap E=E$, and $E$ is the desired arc $C$, 
as $E$ is then the unique subarc of $E$ the endpoints of which go under $g$ into $g(\bar{U})$ and $g(\bar{V})$.

Suppose $Y$ is an indecomposable tree-like continuum whose nondegenerate proper subcontinua are arcs and $f$ is a 2-to- 1 map from a continuum $X$ onto $Y$. Then an $\operatorname{arc} L$ in $Y$ will be said to be trivial with respect to $f$ if $f^{-1}(L)$ splits into two components each of which is mapped onto $L$ (in fact mapped homeomorphically). In view of the theorem of Gottschalk [2], an $\operatorname{arc} L$ will be trivial with respect to $f$ if each component of $f^{-1}(L)$ maps onto $L$.

Lemma 8. Suppose $Y$ is an indecomposable tree-like continuum whose nondegenerate proper subcontinua are arcs, and $f$ is a 2-to-1 map from a continuum $X$ onto $Y$. Then every arc in $Y$ is trivial with respect to $f$.

Proof. Let $L$ be an arc in $Y$. Assume to the contrary that $C$ is a component of $f^{-1}(L)$ such that $f(C) \neq L$. Let $x$ be an endpoint of $L$ such that $x \in f(C)$. For the other endpoint $t$ of $L$, we have $t \notin f(C)$ by assumption.

Extend $L$ through $x$ to a larger $\operatorname{arc} N$, i.e. an arc with endpoint $t$ that contains $L$. Let $y$ be the endpoint of $N$ distinct from $t$. Since the arc components of $Y$ are dense in $Y, x$ is a free endpoint of $L$ (by Lemma 3), and the set of open values of $f$ is open in $Y$ (see a preliminary remark), we shall assume that the endpoint $y$ of $N$ is an open value of $f$.

From Lemma 2 it follows that there exists a component $D$ of $f^{-1}(N)$ such that $t \notin f(D)$. By Lemma 4, another component $F$ of $f^{-1}(N)$ is mapped onto $N$ under $f$. Note that $F$ is the only component that maps onto $N$. It follows that one of the two points that map to $y$, say $a$, belongs to $D$ and the other, say $b$, belongs to $F$. Let $V$ and $W$ be open sets such that $D \subseteq V, W$ contains the other components of $f^{-1}(N)$ (including $F$ ), $\bar{V} \cap \bar{W}=\emptyset$, and $t \notin f(\bar{V})$.

Let $U$ be an open neighborhood of $y$, an open value of $f$, such that $f^{-1}(U)$ splits into two open sets each of which is homeomorphically mapped by $f$ onto $U$. If a point $y^{\prime}$ lies in $U$, then $f^{-1}\left(y^{\prime}\right)$ has points in both of these open sets. Assume, moreover, that $U$ is so small that the two points of $f^{-1}\left(y^{\prime}\right)$ lie in distinct sets $V$ and $W$.

From Lemma 7 we know that there is, for each $\varepsilon>0$, an arc $N^{\prime}$, lying in the same arc component of $Y$ as $N$, with reverse orientation within $\varepsilon$ of $N$. We may choose $\varepsilon$ small enough that the first point, $y^{\prime}$, of $N^{\prime}$ lies in $U$ (and thus $f^{-1}\left(y^{\prime}\right)$ has points in both $V$ and $\left.W\right)$, that $f^{-1}\left(N^{\prime}\right) \subseteq W \cup V$, and that for the other endpoint $t^{\prime}$ of $N^{\prime}$, we have $t^{\prime} \notin f(\bar{V})$. Let $D^{\prime}$ denote a component of $f^{-1}\left(N^{\prime}\right)$ having points in $V$, and let $a^{\prime}$ and $b^{\prime}$ denote the points of $f^{-1}\left(y^{\prime}\right)$ in $V$ and $W$ respectively. Note that exactly one component 
$F^{\prime}$ of $f^{-1}\left(N^{\prime}\right)$ maps onto $N^{\prime}$, and $F^{\prime}$ must contain $b^{\prime}$ and lie in $W$, since both inverse points of $t^{\prime}$ lie in $W$.

Let $M$ be the arc from $y$ to $y^{\prime}$. Consider the arc $S=N \cup M \cup N^{\prime}$. By Lemma 4, one of the components of $f^{-1}(S)$, say $A$, maps onto $S$. The following is a list of the properties of $f$ restricted to $f^{-1}(S)$ :

(a) The component $A$ contains components of $f^{-1}(N), f^{-1}(M)$, and $f^{-1}\left(N^{\prime}\right)$ that are mapped onto the corresponding $\operatorname{arcs} N, M$, and $N^{\prime}$. To see this, apply Lemma 4 to the function $f$ restricted to $A$ mapping into $S$. Thus $A$ contains $F \cup F^{\prime}$ and hence the points $b$ and $b^{\prime}$ that map to $y$ and $y^{\prime}$. Since $A$ is connected, some component of $f^{-1}(M)$ contains $b$ and $b^{\prime}$.

(b) By the theorem of Gottschalk [2], the counterimage $f^{-1}(M)$ is not connected. Hence there is a component $E$ of $f^{-1}(M)$ that does not contain either $b$ or $b^{\prime}$ and must contain either $a$ or $a^{\prime}$.

(c) By assumption, the counterimage $f^{-1}(N)$ has a component $D$ containing $a$ such that $t \notin f(D)$ (and there is only one such component).

(d) The counterimage $f^{-1}\left(N^{\prime}\right)$, of the arc $N^{\prime}$ close to $N$, has a component $D^{\prime}$ containing $a^{\prime}$ such that $t^{\prime} \notin f\left(D^{\prime}\right)$ (and there is only one such component). Recall that $t^{\prime} \notin f(\bar{V})$ and $D^{\prime} \subset V$.

In view of properties (a)-(d), we infer that there exists a component of $f^{-1}(S)$ whose image contains neither of the points $t$ and $t^{\prime}$. This is a contradiction to Lemma 1. To find such a component, consider two cases:

1. E contains exactly one of $a$ and $a^{\prime}$, say $a$,

2. E contains both of $a$ and $a^{\prime}$.

In the first case, $E \cup D$ is such a component, and $E \cup D \cup D^{\prime}$ in the second case.

\section{Theorem and corollary}

THEOREM 1. Suppose that $Y$ is a tree-like continuum whose nondegenerate proper subcontinua are arcs. Then there does not exist a 2-to-1 map from any continuum onto $Y$.

Proof. First note that if $Y$ is an arc, then the theorem of Gottschalk [2] implies that $Y$ is not the 2-to-1 image of any continuum. If $Y$ is not an arc, then our lemmas apply.

In the paper [5] of one of the authors, a map $f: X \rightarrow Y$ between continua is called crisp if the counterimage of each proper subcontinuum $K$ of $Y$ splits into two continua, each of which is mapped homeomorphically onto $K$ and it is proved there (Theorem 1 ) that each crisp map is a local homeomorphism. Our Lemma 8 states that any 2-to- 1 map from a continuum onto $Y$ is crisp. Thus any such map is a homeomorphism since any local homeo- 
morphism from a continuum onto a tree-like continuum is a homeomorphism (Maćkowiak [7]). This contradiction completes the proof.

Subcontinua of a continuum satisfying the hypotheses of the theorem (a tree-like continuum whose nondegenerate proper subcontinua are arcs) also satisfy the hypotheses. Thus the following corollary applies to some finite unions of these continua:

Corollary 1. Suppose that the tree-like continuum $K$ is the union of a finite number of continua, $K_{1} \cup \ldots \cup K_{n}$, such that for each $i$, no subcontinuum of $K_{i}$ is the 2-to-1 image of a continuum. Suppose further that each $K_{i} \cap K_{j}$ is, at most, a single point if $i \neq j$. Then $K$ is not the 2-to-1 image of a continuum.

Proof. Suppose there is a 2-to-1 map from a continuum onto $K$ and suppose $K^{\prime}$ is a subcontinuum of $K$ minimal with respect to having connected preimage. By Theorems 1 and 3 in Fox [1] (or Reference Theorem in [4]), $K^{\prime}$ has no cut point. So $K^{\prime}$ is contained in a single $K_{i}$ since a local cut point in a tree-like continuum is a cut point. But this contradicts the hypothesis that no subcontinuum of $K_{i}$ is the 2-to-1 image of a continuum.

\section{References}

[1] D. Fox, k-to-1 continuous transformations, Dissertation, Univ. of California at Riverside, 1973.

[2] W. H. Gottschalk, On k-to-1 transformations, Bull. Amer. Math. Soc. 53 (1947), 168-169.

[3] J. Grispolakis and E. D. Tymchatyn, Continua which are images of weakly confluent mappings only (I), Houston J. Math. 5 (1979), 483-501.

[4] J. Heath, Tree-like continua and exactly k-to-1 functions, Proc. Amer. Math. Soc. 105 (1989), 765-772.

[5] —, 2-to-1 maps with hereditarily indecomposable images, ibid. 113 (1991), 839-846.

[6] W. T. Ingram, An atriodic tree-like continuum with positive span, Fund. Math. 77 (1972), 99-107.

[7] T. Maćkowiak, Semiconfluent mappings and their invariants, ibid. 79 (1973), 251-264.

[8] S. B. Nadler, Jr. and L. E. Ward, Jr., Concerning exactly $(n, 1)$ images of continua, Proc. Amer. Math. Soc. 87 (1983), 351-354.

INSTITUTE OF MATHEMATICS

SILESIAN UNIVERSITY

BANKOWA 14

40-007 KATOWICE, POLAND
MATHEMATICS DEPARTMENT PARKER HALL AUBURN UNIVERSITY ALABAMA 36849-5310 